\title{
QSO Strong Gravitational Lensing and the Detection of Dark Halos
}

\author{
Andrea V. Macciò \\ Max-Planck-Institute for Astronomy Königstuhl 17, D-69117 Heidelberg, Germany \\ email:maccio@mpia.de
}

\begin{abstract}
We present recent results concerning the possibility to detect dark satellites around galaxies using QSO strong gravitational lensing. Combining high resolution hydrodynamical simulations of galaxy formation and analytic studies we show that current QSO observations data do not present any evidence for the existence of such satellites. The amount of substructures predicted by CDM within a galaxy size dark matter halo is too low to explain the observed anomalies in the QSO images flux ratio.

Nevertheless the fluxes of QSO multiple images can be used to constrain the CDM power spectrum on small scales and test different dark matter candidates. We show that a warm dark matter scenario, with an insufficiently massive particle, fails to reproduce the observational data. Our results suggest a lower limit of few $\mathrm{keV}(\sim 10)$ for the mass of warm dark matter candidates in the form of a sterile neutrino, in good agreement with previous results coming from Lyman- $\alpha$ forest and Cosmic Microwave Background analysis.
\end{abstract}

Keywords. galaxies: halos, dark matter, methods: numerical

\section{Introduction}

Cold Dark Matter (CDM) simulations predict many more low mass satellite haloes than are actually observed in the Milky Way (Klypin et al. 1999, Moore et al. 1999, Diemand et al 2004, DMS04 hereafter). It seems that 10-15\% of the mass was left in satellites with perhaps $1-2 \%$ at the projected separations of $1-2$ Einstein radii $\left(R_{e}\right)$ where we see most lensed images (e.g. Mao et al. 2004); this is far larger than the observed fraction of 0.01-0.1\% in observed satellites (e.g. Chiba 2002).

Solutions to this mismatch were proposed in three broad classes: satellites are present but dark if star formation is prevented (Bullock, Weinberg \& Kravtsov 2000), satellites are destroyed due to self-interacting dark matter, or their formation is prevented by changing the power spectrum to something similar to warm dark matter with significantly less power on the relevant mass scales (e.g. Bode et al. 2001). These hypotheses left the major observational challenge of distinguishing dark satellites from non-existent ones. This became known as the CDM substructure problem.

It has been argued that a possible signature of the presence of dark matter substructures can be found in strong gravitational lensing of QSOs (Mao \& Schneider 1998; Metcalf \& Madau 2001). If a distant image source is close to a cusp (from inside) in a caustic curve, three of the images will be clustered together and the sum of their magnifications will be zero (Zakharov (1995), taking the negative parity image to have negative magnification). This relation holds for a wide class of smooth analytic lens models (Keeton et al. 2003); on the other hand all known observed lensed QSOs violate this relation giving rise to the so-called problem of anomalous flux ratio. This has been explained with the presence of cold dark matter substructure within the lensing galaxy's halo. 
By using low resolution simulations of galaxy formation Bradač et al. (2004) claimed that the level of substructure present in simulation produces violations of the cusp relation comparable to those observed. Amara et al. (2004) using high resolution Nbody simulations came to the opposite conclusion and their results were lately confirmed by Mao et al 2004.

We will revise this issue and present recent results based on hydrodynamical cosmological simulations of galaxy formation, where the presence of a dissipative component greatly enhances the surviving probability of satellites, especially close to the center of the galaxy (Macciò et al 2006). We will also extend the analysis to the effect of substructures that are not resolved in current dark matter and hydrodynamical simulations (Macciò and Miranda 2006). Finally we will present a different possible explanation for the QSO anomalous flux ratio given by haloes that are along the line of sight, in between the source and the observer (Miranda \& Macciò 2007). We will analyze in detail the effects of this class of haloes on an unperturbed cusp configuration in a $\Lambda$ CDM model and in $\Lambda$ Warm Dark Matter models with different values for the mass $m_{\nu}$ of the warm dark candidate. We found that WDM models with $m_{\nu}<10 \mathrm{keV}$ fail in reproducing the observed anomalies in the flux ratio of lensed QSO. Our results provide a new and independent constrain on the mass of a sterile neutrino, and they are in good agreement with previous constrains coming from Lyman- $\alpha$ forest and CMB analysis.

\section{Numerical Simulations}

The hydrodynamical simulations were performed with GASOLINE, a multi-stepping, parallel TreeSPH $N$-body code (Stadel 2001, Wadsley et al. 2004). We include radiative and Compton cooling for a primordial mixture of hydrogen and helium. The star formation algorithm is based on a Jeans instability criteria (Katz 1992). The code also includes supernova feedback as described by (Katz 1992), and a UV background. We run a high resolution cosmological simulation of a Galactic mass halo $\left(M_{d m} \approx 10^{12} \mathrm{M}_{\odot}\right)$ in a concordance $\left(\Lambda=0.7, \Omega_{0}=0.3, \sigma_{8}=0.9\right)$ cosmology. The mass per particle of the dark matter and gaseous particles are respectively $m_{d}=1.66 \times 10^{6} M_{\odot}$ and $m_{g}=3.28 \times 10^{5} M_{\odot}$ and there are about $10^{6}$ particles for each component (dark and gas) in the high resolution region. The same object has also been simulated with dark matter only using the same spatial and mass resolution as the hydro run, we will refer to the two simulations as hydro and $d m$ respectively.

\subsection{Subhaloes properties}

Within the virial radius of the high resolution CDM simulations we can resolve several hundreds of substructure haloes (bound over-dense clusters of particles). We identify subhaloes with SKID (Stadel 2001) and we included in our study only halos with $M>$ $2 \times 10^{8} \mathrm{M}_{\odot}\left(\right.$ i.e. $\left.N_{d m}>100\right)$.

The effect of baryons on the satellites distribution can be easily seen in Figure 1, where we plot the ratio of the number of subhaloes in the hydro and $d m$ simulations. as function of the distance from the center of the galaxy. At $70 \mathrm{kpc}$ we see an increase by a factor of two in the numbers of surviving satellites. Within the inner $40 \mathrm{kpc}$ the number of satellites with mass greater than $2 \times 10^{8} \mathrm{M}_{\odot}$ is enhanced by a factor $4-5$ over that found in the pure dark matter simulation. This excess of satellites close to the center of the main galaxy, can have interesting consequences on the image properties of multiple lensed QSO. 

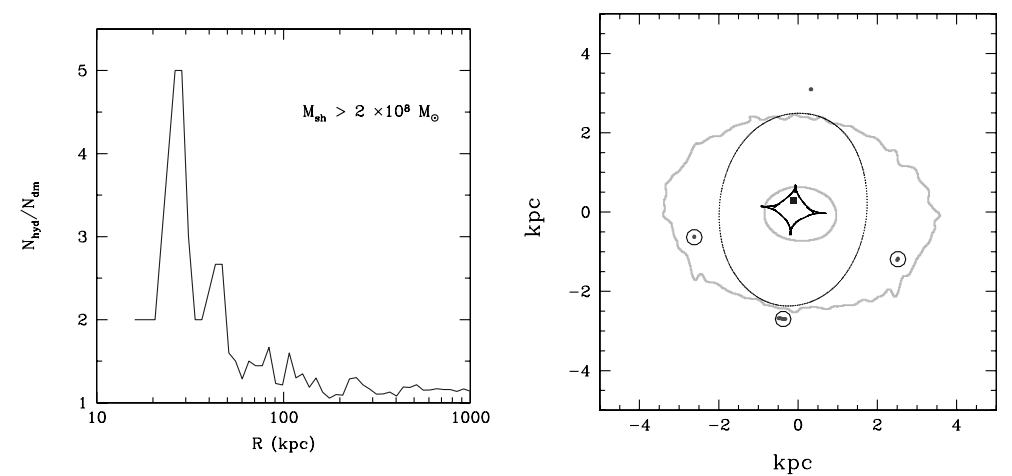

Figure 1. Left:Ratio between the number of DM subhaloes with $M>2 \times 10^{8} \mathrm{M}_{\odot}$ in the hydro and $d m$ runs as function of the distance from the center of the main halo. Right: Basic lens configuration. The caustic surface is shown as a black line and critical curves are shown as cyan lines. The images are shown as red dots, and the three used in the cusp relations are inside black circles. The blue square is the source position.

\subsection{Lensing Analysis and the Cusp configuration}

In order to study the lensing properties of our galaxies we used the Ray Shooting Code described in Macciò (2005). We center our galaxy in a cube of $0.6 \mathrm{Mpc}$ side length and study three lenses, obtained by projecting the particle positions along the coordinate axes. We then divide the projected density field $\Sigma$ by the critical surface mass density for lensing $\Sigma_{c r}=\frac{c^{2}}{4 \pi G} \frac{D_{S}}{D_{L} D_{L S}}$ so obtaining the convergence $k$. Here $c$ is the speed of light, $G$ is the gravitational constant, while $D_{L}, D_{S}, D_{L S}$ are the angular-diameter distances between lens and observer, source and observer, lens and source, respectively. In the following, we adopt $z_{L}=0.3$ We start to compute the deflection angle $\vec{\alpha}(\vec{x})$ on a regular grid of $4096 \times 4096$ test rays, then we propagate a bundle of $16384 \times 16384$ light rays and determine the deflection angle on each light ray by bicubic interpolation amongst the four nearest test rays. The deflection angle diverges when the distance between a light ray and a particle is zero. To avoid this unwanted feature we introduce a softening parameter, $\epsilon_{g}$ and its value is tuned to the resolution of the current simulation. The sources are modeled as circles with a radius of $60 \mathrm{pc}$. A typical lens configuration is shown in Figure 1 (right panel).

There are basically three configurations of four-image systems: fold, cusp, and cross. We will mainly concentrate on the cusp configuration, that corresponds to a source located close to the cusp of the inner caustic curve. The behavior of gravitational lens mapping near a cusp is described by the Cusp relation that states that the sum of the signed magnification factors of the three merging images approaches zero as the source moves towards the cusp. In other words (e.g. Zakharov 1995):

$$
R_{\text {cusp }}=\frac{\mu_{A}+\mu_{B}+\mu_{C}}{\left|\mu_{A}\right|+\left|\mu_{B}\right|+\left|\mu_{C}\right|} \rightarrow 0, \text { for } \quad \mu_{t o t} \rightarrow \infty
$$

where $\mu_{t o t}$ is the unsigned sum of magnifications of all four images, and A,B \& $\mathrm{C}$ are the triplet of images forming the smallest opening angle (see figure 1).

\subsection{Effects of substructures}

Because of the finite size (discreteness) of the particles in the simulations there is a significant amount of shot noise in the surface density estimate, which can affects the lensing properties. The usual approach (Bradač et al. 2004, Amara et al. 2004) is to use 
a Gaussian kernel to smooth the surface density. We used a novel approach to remove substructures from the lensing halo and see how this can change the cusp relation. Our removal procedure works as follows: first we identified all bound substructure using SKID, then each particle belonging to any of the subhaloes is rotated around the center of the galaxy using three random Euler angles. We want to emphasize that we do not physically remove any substructures, because this will change the overall properties of the lens: mass, density profile, etc. This procedure allows us to smooth out only the substructures leaving unaltered all the main features of the primary lens. In Figure 2 the density map of the galaxy is shown, with (left panel) and without (right panel) substructures respectively.

The cusp relation defined by equation 2.1 holds when the source is close to the cusp. As soon as the source moves away from the cusp deviations from $R_{\text {cusp }}=0$ are observed, even for the smooth lens model. On the other hand the closer the source is to the cusp, the smaller is the angle spanned from the three images, so in order to take into account also the position of the source in evaluating the cusp relation it is better to define $R$ as:

$$
R=\frac{2 \pi}{\Delta \theta} R_{\text {cusp }}
$$

where $\Delta \theta$ is the opening angle spanned by the two images with positive parity defined from the center of the galaxy. With this new definition of $R$ a set of three images is said to violate the cusp relation if $R>1$. This makes the comparison between simulations and observations much more straightforward.

The differences in the reduced cusp relation violation in the two cases are shown in Figure 3 (left), where we plot the number of sources that violate the reduced cusp relation as a function of the Gaussian smoothing scale $\left(\epsilon_{g}\right)$. For both the lens models, the number of sources that violate equation 2.2 decreases with the smoothing length because the effect of smoothing is to both reduce the impact of substructures and the noise introduced by single particles.

The difference between the two is not so large, with a maximum for $\epsilon_{g}=0.5 \mathrm{kpc}$, where the number of violations grows from $19 \%$ to $23 \%$, this is because this value of $\epsilon_{g}$ is large enough to cancel the Shot noise, but not large enough to smear out the subhaloes in the simulation, in good agreement with the results of Amara et al. For smaller value of $\epsilon_{g}$ the signal is almost completely dominated by Shot noise, and for larger values we smooth too much, losing spatial information on the surface density of the lens. Figure 3 (left) clearly shows that the impact of substructure in a mass range $10^{7}-10^{9}$ is very weak in disturbing the cusp relation.

Nevertheless a tentative comparison with observation can be made; there are 5 observed cusp caustic lenses systems: B0712+472 (Jackson et al 1998), B2045+265 (Koopmans et al 2003), B1422+231 (Patnaik \& Narasimha 2001), RXJ1131-1231 (Sluse et al. 2003) and RXJ0911+0551 (Keeton et al 2003); the first three are observed in the radio band, the last two in optical and IR. Three of them violate the reduced cusp relation (i.e. $R_{\text {cusp }}>\Delta \theta / 2 \pi$ ). This means a $60 \%$ violation, that is significantly larger than the 15 $25 \%$ we found for our simulations. The sources size used in this work (60pc) allows us to make a direct comparison mainly with QSO observed in radio than in optical or IR, even in this case we have a violation of the reduced cusp relation in 2 objects over 3 , that means $66 \%$ violation. Figure 3 (right) shows the distribution of the values of the reduce cusp relation $R$ both for data and simulates systems (with $\epsilon_{g}=0.5 \mathrm{kpc}$ ). Simulation results are unable to reproduce the high value tail that arises in the observational data; again it is possible to see that the effect of subhaloes is very weak in disturbing the cusp relation, and they only marginally enhance the number of systems with $R>1$. 

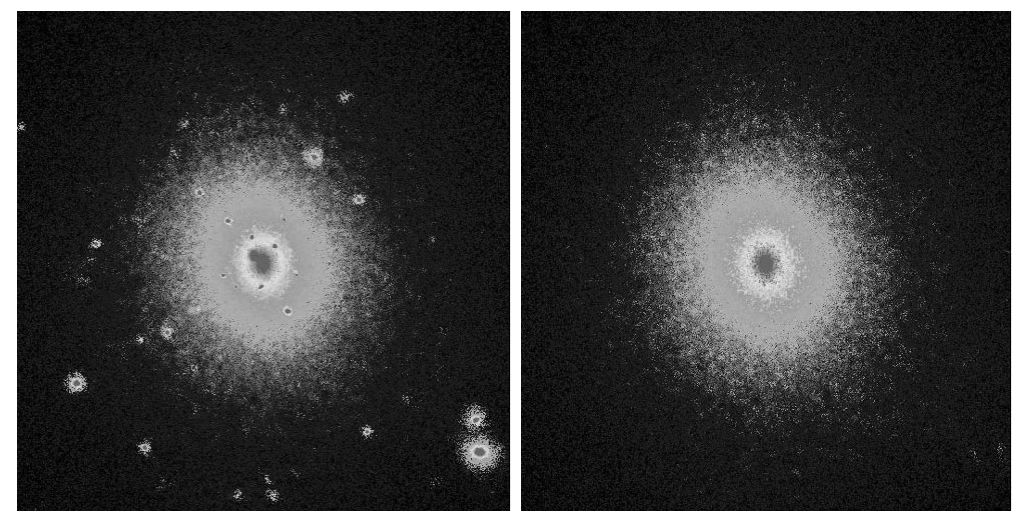

Figure 2. Left: Density map of the mass distribution within the full hydrodynamical simulation. The size of the box is $200 \mathrm{kpc}$. Right: smoothed mass distribution after randomizing the positions of the particles within the substructure haloes
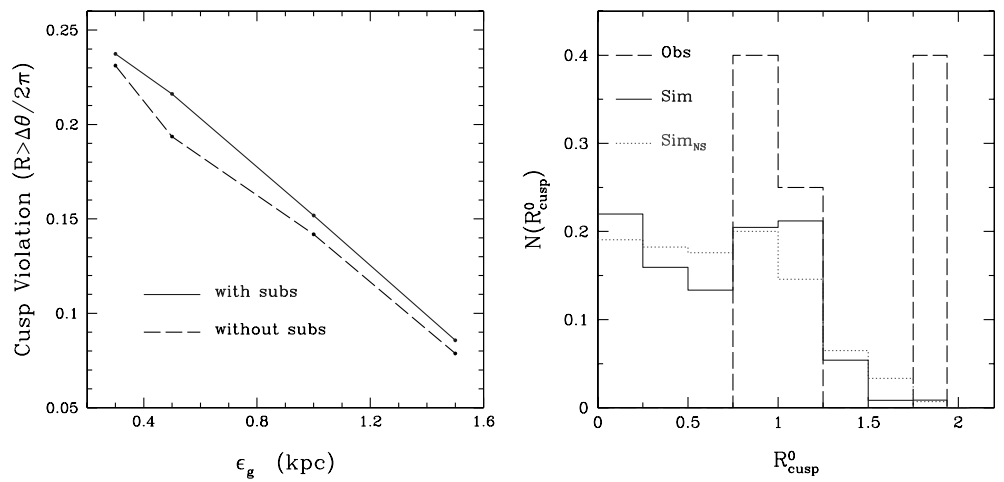

Figure 3. Left:Fraction of total number of sources violating the cusp relation as a function of the Gaussian smoothing length $\epsilon_{g}$. Solid line is for the whole galaxy, dashed line for the galaxy without substructures (see text for its definition). Right: Distribution of $R$ values. The solid and dotted lines show the simulation results before and after the substructure removal. The long histogram line represents the observational data.

\section{Haloes beyond numerical resolution}

As already discussed above, the difference between data and simulation results can arise from the current resolution limitations in the Numerical simulations. In this section we will try to better quantify the effects on QSO lensing of haloes that are not resolved in numerical simulations.

In order to solve the lens equation, we use the LENSMODEL package (Keeton 2001) modeling the main lens galaxy as a singular isothermal ellipsoid (SIE) and the substructures as NFW (Navarro, Frenk \& White 1997) haloes. First, using the gravlens task, we find three lens configurations for which the cusp relation is roughly satisfied. As second step a variable number of substructures is added to the main lens. For this new lensing system (main lens plus subhaloes) we compute again positions and fluxes of the images obtaining a new value for the cusp relation $R$. This procedure is repeated more than 20.000 times for each studied cusp configuration. This allows us to compute the probability distribution of the $R$ value in presence of subhaloes (i.e. figure 4, right panel). 


\subsection{Subhaloes distribution}

We focus our attention only of those subhaloes that are not resolved in current hydro/Nbody simulations In order to evaluate the number density substructures in the mass range $10^{5}-10^{7} \mathrm{M}_{\odot}$ we have made some extrapolations based on results from high resolution N-body simulations. The mass function of subhaloes inside the virial radius of an halo is close to a power law (DMS04) $N(>m) \propto m^{-\beta}$ with a slope $\beta \approx 1$, so that we expect to have a factor $\approx 100$ more subhaloes inside the viral radius if we move our mass threshold from $10^{7} \mathrm{M}_{\odot}$ to $10^{5} \mathrm{M}_{\odot}$.

As said in the previous section such small haloes will affect the $R$ relation only if their distance from the images is of the same order or smaller than the distance between the images themselves. Therefore we need an estimation of the number of haloes inside a small area surrounding the images. This number will also depend on the distance of our area from the center, due to the fact that the number density of haloes increases approaching the center of the main halo (primary lens) as clearly shown in fig 4 (left), which is based on numerical simulations of 4 galaxy size haloes (DMS04).

Consequently the number of subhaloes with a mass greater than $m$ inside an area $A$ at a distance $R$ from the center of the galaxy is:

$$
N_{A}(>m, R)=\frac{\left\langle N_{r_{v}}\left(>m_{0}\right)\right\rangle \frac{m_{0}}{m} N(R) A}{\pi r_{v}^{2}},
$$

where $\left\langle N_{r_{v}}\left(>m_{0}\right)\right\rangle$ is the average number density of subhaloes with $m>m_{0}$ (being $m_{0}$ an arbitrary mass value) inside the virial radius $r_{v}$ and $N(R)$ is the radial $2 \mathrm{D}$ number density of satellites at a projected distance $R$ from the center in units of $\left\langle N_{r_{v}}\left(>m_{0}\right)\right\rangle$. These last two quantities can be obtained directly from N-body simulations. We fix $R \approx 1 \operatorname{arcsec}$ and $A=6 \operatorname{arcsec}^{2}$ ( $A_{6}$ hereafter). Using eq. 3.1 and a mass threshold $m=5 \times 10^{5} \mathrm{M}_{\odot}$ the number of subhaloes within $A_{6}$ ranges from 4 to 12 (depending on $N(R)$, see figure 4, right panel). For each lensing configuration analyzed in this work we added a random number of substructures between $4-12$ to the primary lens with a random mass generated according to $N(>M)$ in the range $10^{5}-10^{7} \mathrm{M}_{\odot}$. These subhaloes are then placed following the $2 \mathrm{D}$ density profile inside the area $A_{6}$ that encloses the three images (cfr. the (blue) square in figure 4, central panel). We have modeled our subhaloes with an NFW density profile. We have adopted different concentration parameters to mimic the scatter present in the mass-concentration relation (Macciò et al 2007).

\subsection{Results}

The central panel of Figure 4 shows the starting cusp configuration with an unperturbed value of $R=0.09$. For this configuration we have generated 20.000 different lensing systems that include substructures according to eq: 3.1 . In figure 4 (right panel) it is shown the probability distribution for $R$ for different numbers of substructures. The maximum of the probability is obtained for the unperturbed value (0.09) and the tail of the distribution extends to 0.12 , but for a very low number of configurations (less than $1.0 \%)$, it is also possible to note that an increase of the total number of substructures (from 4 to 10) does not substantially change the value of $R$. Results for other initial configurations can be found in Macciò and Miranda 2006.

We want to stress that there is nearly always the possibility to explain an anomalous flux ratio using subhaloes, but that their positions and masses must be tuned in a very precise way (i.e distances between images and subhaloes must be less than 0.08 arcsec). Most important, our Monte Carlo simulations show that the probability of obtaining such a fine tuning is very low. As a consequence we conclude that an explanation for the 

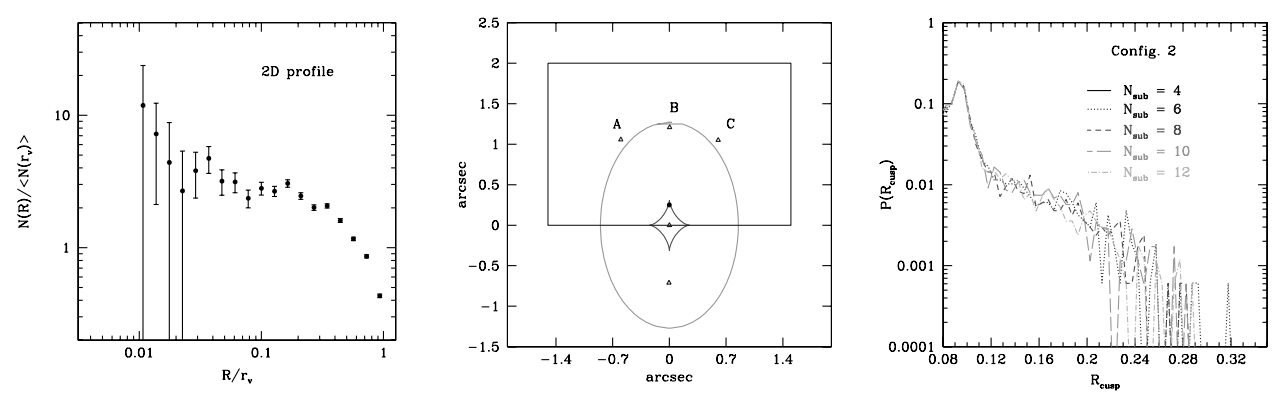

Figure 4. Left:Two dimensional radial number density of subhaloes in units of the average number density inside the virial radius. Center: The unperturbed lens configuration, $(\mathrm{R}=0.09)$. Right: Probability distribution for $R$, for different numbers of substructures inside $A_{6}$.
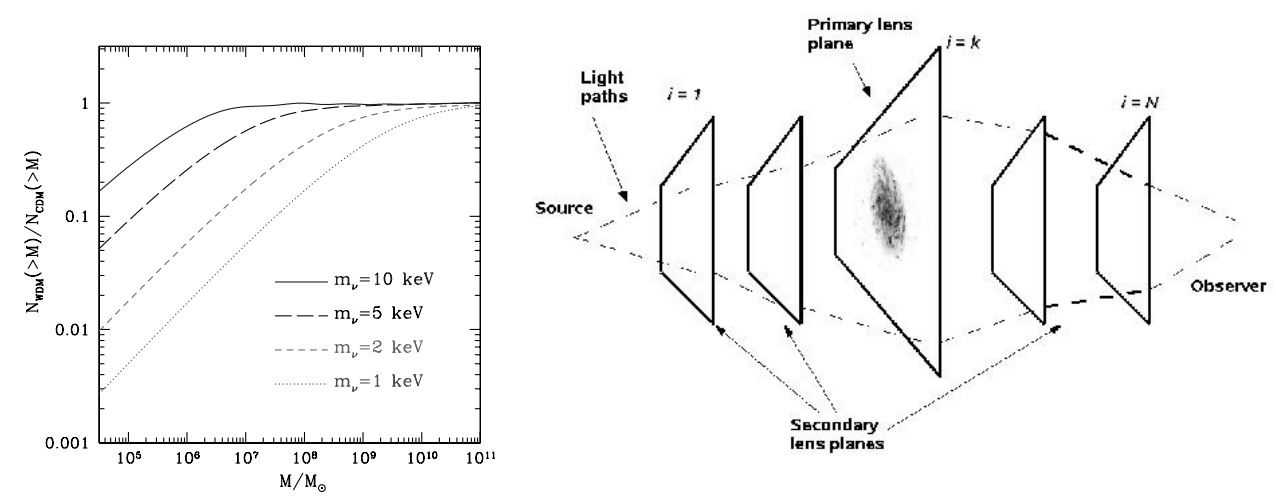

Figure 5. Left:Effects of WDM particles on the dark matter halo mass function at redshift zero. Right: A schematic diagram of the type of multiple plane lensing system being considered

high number of observed anomalous flux ratios in lensed QSOs based on the presence of subhaloes in the mass range we have tested is very unlikely.

\section{Haloes along the line of sight}

The hierarchical formation scenario predicts that the universe should be filled by a large number (more then $10^{3}$ per $h^{-1} \mathrm{Mpc}^{3}$ ) of dark matter haloes with masses $M \approx 10^{6} \mathrm{M}_{\odot}$. Their number density is well described by the Sheth and Tormen mass function (ST: Sheth \& Tormen 2002). In this study we adopted the following values for dark energy and dark matter density, normalization and slope of the matter power spectrum: $\Omega_{\Lambda}=0.74$, $\Omega_{m}=0.26, \sigma_{8}=0.9$ and $n=1$.

The transfer function for the CDM model (and its redshift evolution) has been generated using the public code CMBFAST (Seljak \& Zaldarriaga 1996). For computing the transfer function for WDM models we used the fitting formula suggested by Bode et al. (2001) using for the free parameter $\alpha$ the parameterization of Viel et al. (2005). The main effect of WDM is to dampen the power spectrum of fluctuation on small scales, reducing the number of haloes at low masses. Figure 5 shows the ration between halo number density in WDM and CDM models as a function of the WDM mass $m_{\nu}$. 


\subsection{Lensing simulations}

The main lens parameterization is the same as in 3 and we model our perturbers as singular isothermal spheres (SIS). For the $10^{6} M_{\odot}$ haloes relevant for this work, the SIS profile does not differ dramatically from the NFW profile inferred from cosmological Nbody simulations (Keeton 2003). Besides, the SIS model yields conservative results (see Miranda and Macciò (2007) for a detailed discussion). A SIS halo model is completely characterized by its Einstein radius $\theta_{E}$. We filled the portion of Universe along the line of sight with cubes, then the matter inside each cube is projected onto the middle plane (see figure 5). We used a total of 100 different lens planes roughly equally distribute in space between the source and the observer. This results in $N_{1}=85$ planes behind the main lens and $N_{2}=15$ planes in front of it.

We populated each cube with dark matter haloes, their total number and their mass distribution has been chosen accordingly to the ST mass function at the appropriate redshift. Haloes positions have been randomly assigned. Within a solid angle $d \Omega$ of 3 " $\times 3$ " squared arcsec, the total number of haloes with mass larger than $10^{6} \mathrm{M}_{\odot}$ results to be 512 for the $\Lambda \mathrm{CDM}$ model adopted. This number drops in a consistent way in a warm dark matter scenario, depending on $m_{\nu}$. For a WDM particles mass of $10 \mathrm{keV}$ we obtain 238 haloes along the line of sight within the same $d \Omega$, and even less $(156,135$ for a less massive choice for $m_{\nu}(7.5,5 \mathrm{keV}$, see fig 5 , left panel).

On each single lens plane the total effect on the image magnification factor $\mu$ is obtained by summing up contributions by each perturber. For the source, we adopt the point-like approximation (see Miranda and Macciò (2007) for a detailed discussion on the different approximations adopted in this study).

In a single realization of our perturbed lens configuration the light coming from the source is deflected by $\approx 500$ haloes (plus the main lens) before reaching the observer. Each one of the three images forming the cusp configuration is shifted and amplified, giving as a result a modified $R$ value, different from the original (unperturbed) one of $R_{\text {cusp }}=0.09$. In total we performed 2,000 realizations (with different random seeds for generating masses and positions of perturbers) of each model (CDM/WDM), obtaining 2,000 final different lensing configurations.

\subsection{Results}

Figure 6 (right panel), shows the $R$ probability distribution in the standard $(\Lambda) \mathrm{CDM}$ model and in a WDM model with a sterile neutrino mass of $12.5 \mathrm{keV}$, which is close to the current limit provided by Lyman- $\alpha+$ CMB analysis (Seljak et al. 2006). The signal coming from haloes along the l.o.s. has a probability distribution which remains almost flat in $R$ range 1-2, where 2 (over 5 ) of the observed systems lay. Thanks to this pronounced tail at high $R$ values, in both the warm and cold dark matter scenario, haloes along the line of sight can easily account for the two observed cusp systems with $R \approx 2$, offering a viable solution to the anomalous flux ration issue.

On the contrary a warm dark matter model with less massive particle (i.e. with a higher free streaming scale length) fails in reproducing the observational data due to the reduce number density of haloes along the line of sight as shown in figure 6 , central panel. Changing the WDM particle mass from $m_{\nu}=12.5$ to $m_{\nu}=7.5 \mathrm{keV}$, the tail at $R=2$ drops from a $10 \%$ probability to a $1.5 \%$ one. For $m_{\nu}=5 \mathrm{keV}$ we have a $P(R)$ higher than $5 \%$ only for $R<1.3$.

Finally Figure 6 (right panel) shows the $R$ probability distribution for the three possible categories of perturbers analyzed so far. The dotted (red) line shows the effect of massive subhaloes $\left(M>10^{7} \mathrm{M}_{\odot}\right)$ inside the primary lens (see section 2). The short-dashed (cyan) line shows the effect of lower mass subclumps (still inside the primary lens) as measured 

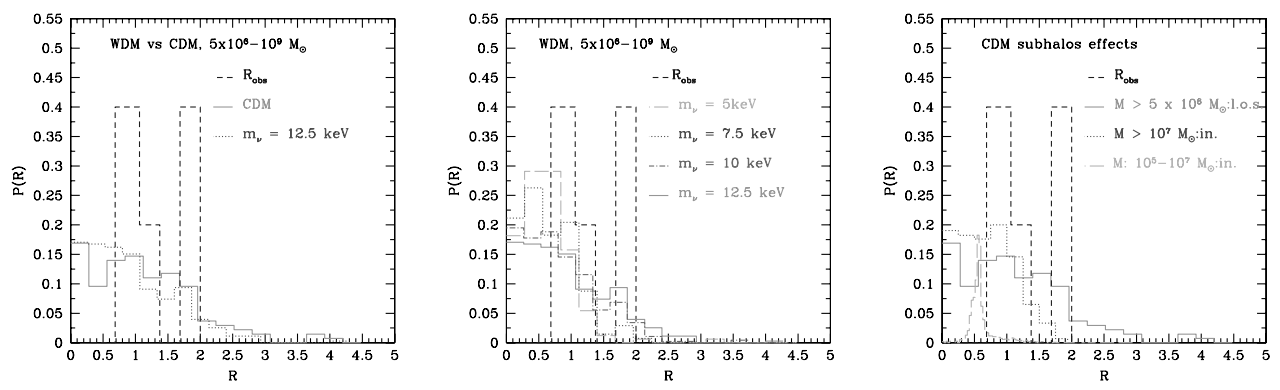

Figure 6. Left: $R$ distribution probability for: observed values (dashed line), CDM haloes $M>5 \times 10^{6} M_{\odot}$ (solid line) and WDM subhaloes with $m_{\nu}=10 \mathrm{keV}$ (dotted line). Center: $R$ probability distributions for different warm particle masses. Right: $R$ probability distribution for different categories of (sub)haloes within the CDM scenario: substructures with $M>10^{7} \mathrm{M} \odot$ (dotted line), subhaloes with $M=10^{5}-10^{7} \mathrm{M}_{\odot}$ (cyan line) and haloes along the line of sight with mass $>5 \times 10^{6} \mathrm{M}_{\odot}$ (green solid line). Observation are shown as a blue long dash histogram.

in section 3. The solid (blue) line shows the effect of the haloes along the line of sight considered in this section in a $\Lambda$ CDMmodel. As already noticed the first two categories of perturbers fail in reproducing the high value tail that arises in the observational data around $R=2$.

\section{Discussion and Conclusions}

The satellites abundance around a galaxy size dark matter haloes is a challenging problem for the Cold Dark Matter scenario. If those haloes exist then they should be dark and the then gravitational lensing is a powerful tool to detect them.

The observed anomalous flux ratio in lensed QSO can be explained by adding small perturbations to the smooth model use to parameterise the main lens. Those perturbers can be identified with dark matter haloes that happen to be close to the images light path. Using recent results based on hydrodynamical simulations we have shown it is hard to reconcile the observed high number of cusp relation violations with the total amount of substructures inside the primary lens predicted by the $\Lambda$ CDM model. This is true even when the limited mass resolution of numerical simulations is taken into account. So we conclude that there is not a direct evidence for dark haloes coming from QSO lensing and that the anomalous flux ratio cannot be explained by perturbation effects due to substructures inside the primary lens.

On the other hand the hierarchical formation scenario predicts that the universe should be filled by a large number (more then $10^{3}$ per $h^{-1} \mathrm{Mpc}^{3}$ ) of dark matter haloes with masses $M \approx 10^{6} \mathrm{M}_{\odot}$. Employing the Sheth \& Tormen mass function we estimate that the expected number of haloes in this mass range along the line of sight of lensed QSO is of the order of few hundreds. Using direct lensing simulations and singular isothermal sphere approximation we computed the effects of those haloes on an unperturbed cusp configuration. We generated more than $10^{4}$ different realizations of our global (lens + perturbers) lensing system, varying masses, positions and number of haloes.

We found that on a statistical basis (averaging on different realizations) this class of perturbers can modify consistently the fluxes of QSO multiple images at a level comparable to the observed one, in good agreement with previous studies on this subject (Metcalf 2005). An important result of our study is that the bulk of the signal on QSO fluxes is due to haloes in the mass range $10^{6}-10^{7} \mathrm{M}_{\odot}$. Since the number density of such 
haloes, and therefore their effect on the cusp relation, can be strongly dampen by the presence of a WDM candidate, the observed number of anomalous flux ratio can be used to parameterise constrain the mass of WDM particles.

We show that if WDM is due to a sterile neutrino, then, in models with $m_{\nu}<10 \mathrm{keV}$, the number of dark haloes along the line of sight is too low to affect in a consistent way the fluxes of lensed QSO, failing in reproducing the observed abundance of systems with high $R$ values. This lower limit for the mass of the sterile neutrino is in good agreement with results obtained using different methods. Future experiments such as Dune, are likely to observe more then 1000 lensed quasars, of which several hundreds should be quadruples due to the magnification bias. Providing new lensing systems to be analyzed and thus tighter constrain on the WDM scenario.

\section{References}

Amara, A., Metcalf, R. B., Cox, T. J., \& Ostriker, J. P. 2006, MNRAS, 367, 1367

Blandford R. \& Narayan R., 1986, ApJ, 310, 568

Bradač M. et al., 2004, A\&̈A, 423, 797

Bode, P., Ostriker, J. P., \& Turok, N. 2001, ApJ, 556, 93

Bullock J. S., Kravtsov A. V. \& Weinberg D. H., 2000, ApJ, 539, 517

Chiba, M. 2002, ApJ, 565, 17

Diemand J., Moore B. \& Stadel J., 2004, MNRAS, 353, 624

Jackson, N. et al., 1998, MNRAS, 296, 483

Katz, N. 1992, ApJ, 391, 502

Keeton, C. R., Gaudi, B. S., \& Petters, A. O. 2003, ApJ, 598, 138

Keeton, C. 2001, preprint, astro-ph/0112350

Klypin A., Kravtsov A. V., Valenzuela O., Prada F., 1999, ApJ, 522, 82

Koopmans, L. V. E., et al., 2003, ApJ, 595, 712

Macciò A. V., 2005, MNRAS, 361, 1250

Macciò A. V., Moore B., Stadel J., \& Diemand J. 2006, MNRAS, 366, 1529

Macciò A. V. \& Miranda, M. 2006, MNRAS, 368, 599

Macciò A. V., Dutton A. A., van den Bosch F. C., Moore B., Potter D., Stadel J., 2007, MNRAS, 378,55

Mao S., Jing Y., Ostriker J. P., Weller J., 2004, ApJ, 604, L5

Metcalf, R. B. \& Madau, P. 2001, ApJ, 563, 9

Metcalf, R. B. 2005, ApJ, 629, 673

Miranda M. \& Macciò A. V., 2007, MNRAS in press, arXiv:0706.0896

Moore B., Ghigna S., Governato F., Lake G., Quinn T., Stadel J., Tozzi P., 1999, ApJ, 524, L19

Navarro J. F., Frenk C. S., White S. D. M., 1996, ApJ, 462, 563

Patnaik, A. R. \& Narasimha, D. 2001, MNRAS, 326, 1403

Seljak, U., Makarov, A., McDonald, P., \& Trac, H. 2006, Phys. Rev. Lett., 97, 191303

Sluse, D. et al., 2003, A\&̈A, 406, L43

Viel, M., Lesgourgues, J., Haehnelt, M. G., Matarrese, S., \& Riotto, A. 2005, Phys. Rev. D., 71, 063534

Stadel J., 2001, PhD thesis, U. Washington

Wadsley, J. W., Stadel, J., \& Quinn, T. 2004, New Astron., 9, 137

Zakharov, A. F. 1995, A\& A, 293, 1 